\title{
Ações de prevenção e enfrentamento das IST/AIDS vivenciadas por mulheres encarceradas
}

\author{
Actions to prevent and cope with the STI/AIDS experienced by women in prison \\ Acciones de prevención y enfrentamiento a las IST/SIDA vivenciadas por mujeres encarceladas
}

\author{
Márcio Pereira Lôbo'; Lucia Helena Garcia Penna"; Joana labrudi Carinhanha"ll; Alba Benemérita Alves Vilela"V; \\ Sérgio Donha Yarid"; Charles Souza Santos ${ }^{V I}$
}

\begin{abstract}
RESUMO
Objetivo: analisar ações de prevenção e enfrentamento das IST/AIDS em mulheres encarceradas, considerando as dimensões de vulnerabilidade. Método: pesquisa qualitativa com aporte teórico-metodológico da Narrativa de Vida de Bertaux. Teve como cenário de estudo o Conjunto Penal de Jequié-BA. Os dados foram coletados através da entrevista aberta com 15 mulheres encarceradas. Estudo aprovado POR Comitê de Ética em Pesquisa. Dados tratados por meio da análise temática de Bertaux Resultados: a utilização de preservativo, seguindo critérios pessoais e culturais, e acesso aos serviços de saúde e insumos proporcionados pelo presídio constituem ações preventivas que as mulheres não teriam fora dele. Contudo, práticas sexuais desprotegidas prevalecem sobre a decisão em utilizar medidas protetivas, pois é fortemente determinada pela cultura e hábitos pregressos, bem como pela dinâmica do presídio. Conclusão: as ações de prevenção podem reduzir a vulnerabilidade das mulheres encarceradas, porém medidas individuais e institucionais voltadas para estas práticas preventivas efetivas permanecem como desafio dentro do sistema prisional.
\end{abstract}

Descritores: Saúde da mulher; prisões; doença sexualmente transmissível; prevenção de doenças.

\section{ABSTRACT}

Objective: to examine STI/AIDS prevention and coping measures for women in prison, considering the dimensions of vulnerability. Method: this qualitative study drew theoretically and methodologically on Bertaux's Life Narrative. The study setting was the penal facility at Jequié, Bahia. Data were collected through an open interviews of 15 women inmates. The study was approved by the research ethics committee. Data were analyzed by Bertaux thematic analysis. Results: condom use, on personal and cultural criteria, and access to health services and materials provided by the prison are preventive measures that women would not have on the outside. Nonetheless, unprotected sex practices prevail over the decision to use protective measures, which is strongly determined by culture and past habits, as well as by the dynamics of the prison. Conclusion: preventive measures can reduce vulnerability of women in prison, but individual and institutional measures directed to these effective preventive practices remain a challenge within the prison system.

Descriptors: Women's health; prisons; sexually transmitted disease; disease prevention.

\section{RESUMEN}

Objetivo: analizar acciones de prevención y enfrentamiento de las IST/SIDA en mujeres encarceladas, considerando las dimensiones de vulnerabilidad. Método: investigación cualitativa con aporte teórico-metodológicamente de las Narrativa de Vida de Bertaux. El escenario de estudio fue el Conjunto Penal de Jequié-BA. Los datos han sido recolectados a través de la entrevista abierta con 15 mujeres encarceladas. Estudio aprobado por el Comité de Ética en Investigación. Los datos han sido tratados por el análisis temático de Bertaux. Resultados: la utilización del preservativo, siguiendo criterios personales y culturales, y el acceso a los servicios de salud e insumos proporcionados por la prisión, constituyen acciones preventivas que las mujeres no tendrían fuera de ella. Sin embargo, las prácticas sexuales desprotegidas prevalecen por encima de la decisión de utilizar medidas protectoras, pues son fuertemente determinadas por la cultura y los hábitos pasados, así como por la dinámica de la cárcel. Conclusión: las acciones de prevención pueden reducir la vulnerabilidad de las mujeres encarceladas, pero medidas individuales e institucionales volcadas a estas prácticas preventivas efectivas permanecen como desafío dentro del sistema penitenciario.

Descriptores: Salud de la mujer; prisiones; enfermedades de transmisión sexual; prevención de enfermedades.

\section{INTRODUÇÃo}

O número de mulheres encarceradas é muito elevado no Brasil. O país é a quinta maior população carcerária do mundo com 37.380 detentas em prisões estaduais e federais, estando apenas atrás dos Estados Unidos (205.400), da China (103.766) Rússia (53.304) e Tailândia $(44.751)^{1}$.

\footnotetext{
'Enfermeiro. Doutorando. Professor Assistente, Universidade Estadual do Sudoeste da Bahia. Brasil. E-mail: marcioplobo@gmail.com "Enfermeira. Doutora. Professora Adjunta, Universidade do Estado do Rio de Janeiro. Brasil. E-mail: luciapenna@terra.com.br

I'Enfermeira. Doutora. Professora Adjunta, Universidade do Estado do Rio de Janeiro. Brasil. E-mail: iabrudi@yahoo.com

IVEnfermeiro. Doutora. Professora Plena, Universidade Estadual do Sudoeste da Bahia. Brasil. E-mail: albavilela@gmail.com

vodontólogo. Doutor. Professor Titular, Universidade Estadual do Sudoeste da Bahia. Brasil. E-mail: yarid@uesb.edu.br

viEnfermeiro. Doutorando. Professor Assistente, Universidade Estadual do Sudoeste da Bahia. Brasil. E-mail: charlesss@uesb.edu.br
} 
Apesar do número aumentado de mulheres infratoras, o sistema prisional brasileiro não acompanhou a ampliação e adequação da infraestrutura penal para essa população. As mulheres continuam dividindo espaço, mesmo que em blocos separados com homens nos presídios mistos, uma vez que se tem um quantitativo muito pequeno de presídios femininos ${ }^{1-2}$

Atualmente, o Sistema Prisional Brasileiro adota medidas de funcionamento conforme estabelecida pela Lei de Execução Penal $7210 / 84^{3}$ e pela Lei $11.106 / 2005^{4}$ que inseriu modificações no Código Penal Brasileiro principalmente no que se refere as peculiaridades femininas. Contudo, o sistema prisional ainda adota postura androcêntrica, que não valoriza as especificidades das mulheres, colocando-as em condição de vulnerabilidade incrementada ${ }^{5}$.

Do ponto da vista da atenção à saúde, as políticas públicas voltadas para as pessoas privadas de liberdade, bem como aquelas voltadas para as mulheres encarceradas, sinergicamente visam garantir o direito de acesso à saúde, respeitando a cidadania, a qualidade, a integralidade e a igualdade da assistência à saúde, sem preconceitos ou privilégios de qualquer espécie para toda a população privada de liberdade, incluindo a população feminina ${ }^{6-9}$.

São preconizadas ações de promoção da saúde e prevenção de agravos transmissíveis ou não, inclusive, aqueles decorrentes do confinamento, como prevenção do uso de álcool e outras drogas, proteção contra Influenza, Infeç̧ões Sexualmente Transmissíveis e Síndrome da Imunodeficiência adquirida (IST/AIDS), distribuição de preservativos masculino e feminino, promoção e cuidado dos direitos sexuais e reprodutivos, promoção da saúde bucal e acesso aos programas de saúde mental; além das ações de vigilância sanitária, a serem implementadas de forma integrada à rede de saúde do território. Apesar dos esforços das políticas públicas para a ampliação e humanização do atendimento à essas mulheres, as ações ainda são insipientes e não respondem as demandas de atenção à saúde das mulheres presentes nestes recintos ${ }^{10-12}$.

Os espaços precários de confinamento, a não adequação das estruturas do sistema prisional à população feminina e a superlotação favorecem situações de maior vulnerabilidade, tais como violências, insalubridades, sedentarismo, uso de drogas, má alimentação, falta de higiene, assistência à saúde inadequada ou incompleta, práticas sexuais desprotegidas, entre tantas outras ${ }^{14}$.

Dentre os agravos à saúde, as IST/AIDS ganham destaque no que diz respeito à vulnerabilidade das mulheres em situação de prisão, pois se verifica maior vulnerabilidade delas a essas doenças, bem como maior barreira para implementação dos direitos sexuais e reprodutivos nesses espaços ${ }^{10,14-16}$. A mulher que ingressa na prisão tem maior risco de ser portadora de alguma IST, em decorrência de uma maior probabilidade de se envolver com a prostituição e com histórias de abuso sexual em sua vida cotidiana anterior à prisão. É fato que, o sistema carcerário agrava, muitas vezes, tais situações de vulnerabilidade pregressa ${ }^{11,15}$.

Considerando as condições precárias vividas pelas mulheres no cárcere, sua vulnerabilidade às IST/AIDS, mas também a existência e preconização legal de ações e políticas de saúde a serem implementadas no sistema penitenciário, inquieta-nos conhecer, a luz das perspectivas de mulheres encarceradas, quais ações de prevenção e enfrentamento às IST/AIDS são estabelecidas no contexto prisional.

Diante do contexto, objetivamos nesse estudo identificar e analisar ações de prevenção e enfrentamento das IST/AIDS em mulheres em situação de cárcere, considerando as dimensões de vulnerabilidades.

\section{REFERENCIAL TEÓRICO}

No contexto prisional, a vulnerabilidade sexual e reprodutiva feminina é incrementada em decorrência das questões biológicas, desigualdades de gênero, estigma e discriminação social ${ }^{16,17}$. Portanto, para compreender a complexidade dos aspectos envolvidos no processo de prevenção e enfrentamento da vulnerabilidade às IST/AIDS das mulheres privadas de liberdade, foi utilizado o amplo conceito de vulnerabilidade desenvolvido por Ayres ${ }^{18}$.

Em saúde, a utilização do termo vulnerabilidade começou no início dos anos 1980. Esse conceito não busca detectar apenas escores probabilísticos de uma pessoa se expor à doença, mas sim, avaliar a influência interligada das dimensões individuais, sociais e programáticas sobre qualquer pessoa ${ }^{18}$.

A dimensão individual envolve aspectos cognitivos como a necessidade de informações das pessoas sobre determinado agravo e, aspectos comportamentais que engloba características pessoais, como desenvolvimento emocional, percepção de risco e atitudes frente aos mesmos ${ }^{19-23}$. A dimensão social refere-se ao ambiente em que as pessoas estão inseridas e se desenvolvem, tal dimensão analisa e avalia o quanto as diretrizes governamentais e a estrutura jurídica-política favorecem o respeito aos direitos humanos e, como estas diretrizes contribuem para o acesso aos bens e serviços, ao respeito às questões culturais e ao exercício da cidadania ${ }^{18,24}$. Na dimensão programática, ligamse os planos individual e social da vulnerabilidade. Ela pode ser identificada nos níveis de poder federal, estadual e 
municipal ou mesmo nas organizações não governamentais (ONG). Esta dimensão é responsável pelo fornecimento de recursos materiais, informações e suporte de ordens variadas para as pessoas ${ }^{18,21,22}$.

\section{METOdOLOGIA}

Trata-se de uma pesquisa qualitativa baseada na narrativa de vida como referencial teórico-metodológico na perspectiva etnossociológica proposta por Daniel Bertaux ${ }^{23}$.

O estudo foi desenvolvido no Conjunto Penal de Jequié (CPJ), na Bahia. Essa unidade prisional é caracterizada por custodiar presos do sexo masculino e feminino, provisórios e condenados, dando cumprimento às penas privativas de liberdade, em regime fechado e semifechado ${ }^{24}$.

Constituíram como participantes, 15 mulheres encarceradas (M1 a M15), que se encontravam cumprindo pena ou aguardando julgamento no CPJ e que se autodeclarassem praticar ou terem praticado relações sexuais em visitas íntimas internas ou externas. Definiu-se como critérios de exclusão: mulheres que apresentavam diagnóstico prévio (pela saúde mental) de desorientação espaço-temporal e/ou com déficit intelectual.

Para a realização da coleta de dados fez-se necessário um período de ambientação do pesquisador no cenário de pesquisa ${ }^{23}$, que durou aproximadamente 6 meses e foi realizado através de conversas informais com os profissionais sobre o funcionamento e cotidiano do presídio e por ações educativas em saúde com as mulheres encarceradas. Posteriormente, as mulheres foram convidadas a participar da pesquisa, expondo os objetivos e método a ser utilizado.

Das 45 mulheres encarceradas no CPJ, 20 aceitaram a participar do estudo, no entanto houve a transferência de duas mulheres para outro conjunto penal e três desistiram de participar do estudo durante o processo de coleta de dados (julho de 2017 a maio de 2018).

Foram realizadas entrevistas narrativas, individuais, a partir da questão norteadora: conte-me um pouco sobre suas relações sexuais antes e após o encarceramento, as quais foram gravadas em áudio. Os encontros ocorreram conforme agendamento prévio com o setor de segurança e disponibilidade das mulheres, que eram direcionadas a uma sala privativa no módulo de saúde do CPJ.

O material proveniente do campo foi submetido ao método de análise temática proposto por Bertaux ${ }^{23}$. Na fase exploratória, as narrativas das mulheres foram escutadas e lidas (após transcrição), seguindo-se a fase de análise comparativa para identificar os traços comuns e diferentes, a fim de desvelar os contextos envolvidos com o objeto do estudo. Enfim, procedeu-se a categorização, que compreendeu as etapas de codificação, recodificação, agrupamento e construção da categoria analítica: Ações de prevenção e enfrentamento às IST/AIDS vivenciadas por mulheres em situação de cárcere.

Este estudo seguiu a Resolução $466 / 202^{25}$, sendo seu projeto aprovado pelo Comitê de Ética em Pesquisa da Universidade Estadual do Sudoeste da Bahia, com CAAE nㅇ6 64271316.2.0000.0055, no de parecer 1.963.

\section{RESULTADOS E DISCUSSÃO}

As ações de prevenção e enfrentamento das IST/AIDS em mulheres encarceradas estão relacionadas as suas condutas sexuais (uso de preservativos), a aquisição de conhecimento quanto a saúde sexual, reprodutiva e IST/AIDS e a oferta de materiais (preservativos) e serviços (consultas e realização de exames) proporcionados pelo serviço público de saúde das unidades de saúde e pelo Programa de Saúde Prisional. Tais ações envolvem as três dimensões da vulnerabilidade ${ }^{20}$.

A prevenção das IST/AIDS pode ser percebida na referência ao uso de preservativo para a prática do sexo protegido, envolvendo a dimensão individual. Apesar de não ser uma rotina nas relações sexuais das mulheres entrevistadas, aparece como uma forma de prevenir IST/AIDS e, sobretudo, gravidez não planejada. Esta conduta preventiva está calcada em diferentes justificativas, que dizem respeito à relação com a parceria sexual, com o seu próprio corpo, com a família/comunidade, ou ainda com o trabalho.

As mulheres entrevistadas tendem a usar preservativo por desconfiança nas condições de saúde do parceiro, seja por ocasião de um relacionamento recente ou de um trabalho (prostituição) ou ainda pelo comportamento masculino tradicionalmente machista de promiscuidade e ou de relações extraconjugais que afirmam sua masculinidade e virilidade dentro ou fora do presídio. Também referiram fazer uso do preservativo por exigência do companheiro nas relações extraconjugais ou em determinados tipos de relação sexual, como no sexo anal. $O$ interesse sexual pela parceria também influencia o uso ou não do preservativo, quer dizer, se não gostam da pessoa, usam preservativo. A utilização de preservativo de forma eventual ou baseada em conjecturas incomuns a prática do sexo protegido foi identificada em outros estudos $26-28$. 
No princípio nós começamos a ter relação, e ele queria sem camisinha. E eu disse não, porque era começo (M12).

Eu não sabia se ele estava só comigo, com outros tipos de mulher [...] só que de um tempo que ele começou a ficar na rua e começou a me visitar, ai eu já não tinha mais a confiança, [...] eu já exigia para a gente voltar a usar camisinha. [...] Mantinha relação com camisinha porque ele era casado (M6).

Eu ficava com ele [na cidade] por interesse das coisas. Nós namorávamos mas não tinha um pingo de graça.(...) com ele eu usava preservativo não sei porque, acho que eu tinha nojo dele (M11).

É estranho você visitar uma pessoa [visita íntima externa] que você nunca viu, nunca vi pela foto só pela voz. E voz ilude. A voz dele era linda, parecia um gato. Quando fui lá ver ... foi um terror. Eu tive relação sexual, ele foi me beijando, foi me chapou. [...] A gente foi com camisinha (M8).

Outra justificativa para o uso do preservativo é a gravidez não planejada. Constata-se também, que a gravidez indesejada, figura como um motivo positivo para diminuir a vulnerabilidade das mulheres IST/AIDS. O medo de ter o corpo modificado pela gestação e da reprovação familiar e social pela gravidez não planejada e ou indesejada evidencia respectivamente, o enfraquecimento de fatores relacionados a vulnerabilidade individual e social para IST/AIDS.

Toda vez era isso, queriam fazer diferente...sem camisinha. Mas eu sempre tive medo de uma gravidez [...] o que dirá minha família? (M5).

Ficava toda besta com meu corpo. Me prevenia para mim não engravidar. Na época eu não sabia de doença sexualmente nenhuma (M14).

Como se pode perceber, esta prática sexual é regida pelas relações de gênero desiguais, realçando a dimensão social da vulnerabilidade às IST/AIDS, que dificulta a sustentação de uma atitude/posição de permanente prevenção. É reconhecido as dificuldades das mulheres em negociar o uso do preservativo ou medo de perder o relacionamento inicial ou estável, portanto, as políticas públicas precisam considerar as questões de gênero a partir das tensões e desigualdade de poder existente nas relações entre homens e mulheres ${ }^{29,30}$.

A condição de ser uma mulher encarcerada, vivendo o cotidiano do sistema prisional com suas normas disciplinares, o acesso à alguns serviços de saúde, tais como as ações educativas, exames preventivos e disponibilização de preservativos, e ainda, o convívio com outros detentos e detentas, influenciou a condição de sua vulnerabilidade (dimensão social e programática) às IST/AIDS. Tais serviços são previstos na Política Nacional de Atenção Integral à Saúde das Pessoas Privadas de Liberdade no Sistema Prisional ${ }^{9}$ e deve ser compreendida como um direito cumprido pelo Sistema que acabam protegendo a população carcerária das IST/AIDS ${ }^{29,30}$.

No que se refere ao conhecimento adquirido quanto a saúde sexual, reprodutiva e IST/AIDS, as mulheres relataram que os conhecimentos foram oriundos dos meios de comunicação como TV e rádio, do aprendizado na escola, das conversas informais, da participação em atividades de educação em saúde realizadas por profissionais de saúde dentro e fora do presídio. Verifica-se que essas práticas são importantes para diminuir a vulnerabilidade destas mulheres a IST/AIDS, pois favorece a construção de conhecimento sobre o funcionamento do corpo e o controle da sua saúde, inclusive, destacam que o ambiente do presídio oportuniza o aprendizado e a instrumentalização para o cuidado à sua saúde mais do que fora dele.

Elas [enfermeiras] foram me ensinando mais, como preservar a saúde, o ambiente. [...] toda vez que eu for.praticar e me relacionar com outro tipo de pessoa, me prevenir e continuar prevenindo; procurar fazer sempre exame, procurar sempre orientação das coisas para poder prevenir doença. Eu conheci sobre pílula, camisinha, injeção em conversa entre amigas (M1).

Quando você está na rua, nem todo mundo procura evitar, se prevenir. E quando você está presa em função das palestras, dos conselhos de saúde, você aprende se prevenir, evitar pegar doença. Nós aprendemos muita coisa na cadeia. A mente antes dela ser presa é uma e depois é totalmente outra. Em tudo, em termo de parceiro, em termo de relação, em termos de saber levar a casa, saber levar o relacionamento, você aprende de tudo um pouco (M13).

No que diz respeito, a oferta de materiais e serviços para a promoção da saúde e prevenção de doenças, as mulheres privadas de liberdade também relataram que recebem insumos (como preservativos e remédios) e atendimento à saúde dentro das possibilidades do serviço prisional ou na rede de atenção do território por meio de consultas e exames voltados para a saúde sexual e reprodutiva (como realização de preventivo, testagens sorológicas para as IST/AIDS, planejamento reprodutivo), acompanhados de orientações em saúde. Neste sentido, também referem que essa assistência à sua saúde é maior dentro do presídio do que as ações ofertadas e utilizadas por estas mulheres quando estavam em liberdade, reduzindo a vulnerabilidade programática das mulheres encarceradas a contaminação por IST/AIDS. 
As camisinhas elas [enfermeiras] dão para prevenir a gente [...] Mas se umas usam e outras não usam, elas não podem fazer nada, mas as camisinhas chegam até o módulo [de saúde do CPJ] (M1).

Na prisão, sempre teve preventivo, exames, certas orientações que eu não tive na rua. [...] a enfermeira fornece o preservativo, a pilula anticoncepcional, as injeções, orientações [...] $E$ os exames de rotina na unidade [prisional] (M2).

[...] teve a campanha aqui do preventivo (...) e a enfermeira tomou o nome das mulheres [...] Eu fui a primeira a botar meu nome. Queria saber como eu estava. Todo ano sempre faço. Ela fala o resultado e chama a gente em particular; fala se não deu alguma coisa ou se der algum tipo doença... começa já encaminhar para fazer o tratamento... a cuidar da gente (M1).

Depois que eu entrei no presídio eu tive mais influência para cuidar da saúde. Se eu tivesse na rua agora eu não tinha procurado médico pra fazer o preventivo não, eu estaria tomando remédio na doida como sempre tomei (M8).

A realização de triagem sorológica por meio de exames laboratoriais entre a mulher e o parceiro é apontada como uma forma de decidirem sobre o uso do preservativo. A existência dessa prática, pode tornar as pessoas mais vulneráveis às IST/AIDS, por não garantir a ausência de doença, devido ao período de janela imunológica das doenças, como também não garante a monogamia nas relações sexuais. Mas também, pode ser considerada como uma ação que diminui a vulnerabilidade individual por meio de ações programáticas ofertadas pelo sistema de saúde ${ }^{18}$.

Eu pedi a ele que fizesse o exame. [...]Ele foi e fez. E no exame não deu nada. Então, voltamos a ter relações sem camisinha. Nenhum dos dois tinha nada (M1).

Cabe destacar nas narrativas o esforço da equipe de saúde da unidade prisional em proporcionar o acesso aos serviços de saúde necessários de diagnóstico e tratamento, incluindo a parceria com a rede de atenção do território, proporcionando uma atenção integral à mulher encarcerada, conforme previsto na PNAISP 8 e nas Diretrizes de Atenção à mulher Presa ${ }^{9}$. Verifica-se ainda o empenho da enfermeira para o cuidado integral à mulher encarcerada, bem como o respeito ao seu direito de decisão sobre seu corpo e sua saúde.

Para prevenção da transmissão das IST/AIDS, considerando a dimensão programática, faz-se necessária a efetivação das políticas públicas responsáveis pelas ações que reduzam a vulnerabilidade às IST/AIDS (acesso à informação/educação, rede de apoio à identificação precoce dos casos e tratamento imediato) e que garantam acesso aos serviços de saúde e sociais necessários, sendo que cada grupo populacional carece de ações específicas de acordo com as características intrínsecas dos mesmos, como no caso das mulheres privadas de liberdade ${ }^{18,31-33}$.

Dessa forma, entende-se que as ações de prevenção proporcionadas pelo presídio diminuem a vulnerabilidade programática às IST/AIDS entre as mulheres encarceradas. Contudo, práticas sexuais desprotegidas prevalecem sobre a decisão em utilizar medidas protetivas, pois é fortemente determinada por cultura e hábitos pregressos que desenham seus roteiros sexuais ${ }^{33}$ e são reproduzidos por meio das condutas sexuais ${ }^{34}$, bem como pela dinâmica de funcionamento do presídio, que "foi criado e desenvolvido por homens e para homens" 5:295.

\section{CONCLUSÃO}

As condutas das mulheres encarceradas ao adotarem o uso do preservativo, mesmo que esporadicamente e, seguindo critérios pessoais, parece indicar uma redução de sua vulnerabilidade individual para IST/AIDS. Além disso, a oferta de preservativo, o acesso facilitado aos serviços de saúde, bem como, as ações educativas ofertadas pelos profissionais de saúde do presídio são apontadas como minimizadores de vulnerabilidade programática às IST/AIDS, quando comparado às condutas sexuais e acesso aos serviços de saúde utilizados por estas mulheres fora do sistema prisional.

Contudo, a prática sexual sem proteção tem relação direta com as histórias de vida das mulheres (anteriores à privação de liberdade) e prevalece sobre a decisão em utilizar medidas protetivas para IST/AIDS, pois é fortemente determinada pela cultura e hábitos cotidianos. Vale ainda ponderar sobre o próprio funcionamento do sistema prisional, cuja estrutura é permeada por normas oficiais ou não que seguem a lógica da opressão de gênero e da discriminação como fator determinante da prática sexual desprotegida. Assim, as mulheres mantêm-se vulneráveis em todas as dimensões, indicando que as ações individuais e institucionais voltadas para a adoção de práticas protetivas para IST/AIDS permanece como desafio dentro do sistema prisional, tanto para enfermeiros e outros profissionais de saúde quanto para os gestores.

Ao ouvir essas mulheres privadas de liberdade, este estudo evidenciou êxitos e lacunas da atenção às mulheres privadas de liberdade, considerando sua vulnerabilidade às IST/AIDS. Nesse sentido, as ações do enfermeiro na promoção da saúde e prevenção de agravos são essenciais para debelar o processo de transmissão de doenças dentro do presídio, bem como para a humanização do cuidado e para o fortalecimento dessas mulheres duplamente punidas. 
As limitações deste estudo referem-se à apresentação da realidade de um presídio específico, restringindo a análise mais ampliada sobre o sistema prisional feminino e exigindo mais estudos que proporcionem maior compreensão e reflexão das necessidades e lacunas da atenção à saúde deste grupo vulnerável, além da relevância da discussão desta temática nos ambientes acadêmicos e profissionais, particularmente na área da Enfermagem.

\section{REFERÊNCIAS}

1. Ministério da Justiça (Br). Departamento Penitenciário Brasileiro. Levantamento Nacional de Informações Penitenciaria InfoPen mulheres - Junho de 2014 [Internet]. Brasília (DF): Ministério da Justiça; 2015. [cited 2018 Feb 20]; Available from: http://189.28.128.100/dab/docs/portaldab/documentos/relatorio_depen.pdf

2. Diuana $V$, Ventura $M$, Simas L, Larouzé B, Correa M. Women's reproductive rights in the penitentiary system: tensions and challenges in the transformation of reality. Ciênc. Saúde coletiva 2016 [cited 2018 Jun 02]; 21(7):2041-50. Available from: http://www.scielo.br/scielo.php?pid=S1413-81232016000702041\&script=sci_arttext\&tIng=en

3. Governo Federal (Br). Decreto-Lei de Execução Penal n. 7. 210, de 11 de julho de 1984. Institui a Lei de Execução Penal e a legislação correlata [Internet]. Brasília (DF). Diário Oficial. República Federativa do Brasil; 12 de jul. 1984. [cited 2018 Feb 20]. Available from: http://bd.camara.gov.br/bd/handle/bdcamara/766

4. Ministério da Justiça (Br). Lei № 11.106, de 28 de março de 2005. Altera os artigos. 148, 215, 216, 226, 227,231 e acrescenta o artigo. 231-A ao Decreto-Lei $n^{\circ} 2.848$, de 7 de dezembro de 1940 - Código Penal e dá outras providências [Internet]. Brasília (DF). Diário Oficial da União; 29 de mar. 2005. [cited 2018 Feb 20]. Available from: http://bd.camara.gov.br/bd/handle/bdcamara/766

5. Lima LR, Pacheco RAS. O encarceramento de mulheres sob a perspectiva da criminologia feminista. Revista Jurídica Direito, Sociedade e Justiça [Resumo expandido]. 2017; 5(1):295-7.

6. Conselho Nacional de Justiça (Br). Cartilha da Mulher Presa [Internet]. 2 ed. Brasília (DF): Departamento de Monitoramento e Fiscalização do Sistema Carcerário e do Sistema de Execução de Medidas Socioeducativas (DMF); 2012 [cited 2018 Feb 20]; Available from: http://www.cnj.jus.br/images/programas/comecar-denovo/publicacoes/cartilha_da_mulher_presa_1_portugues_4.pdf

7. Presidência da República (Br). Secretaria de Políticas para as Mulheres. Plano Nacional de Políticas para as Mulheres 2013-2015 [internet]. Brasília (DF): Secretaria de Políticas para as Mulheres; 2013. [cited 2018 Feb 20] Available from: http://www.spm.gov.br/assuntos/pnpm/publicacoes/pnpm-2013-2015-em-22ago13.pdf

8. Ministério da Saúde (Br). Secretaria de Atenção à Saúde. Política Nacional de Atenção Integral à Saúde das Pessoas Privadas de Liberdade no Sistema Prisional [Internet]. Brasília (DF): Ministério da Saúde; 2014.

9. Governo do Estado (SP). Coordenadoria de Reintegração Social e Cidadania. Diretrizes de Atenção à Mulher Presa [Internet]. São Paulo; 2013. [cited 2018 Feb 20] Available from: http://www.reintegracaosocial.sp.gov.br/db/crsckyu/archives/6208c81fb200c6081c054df541387c7b.pdf

10. Ministério da Justiça (Br). Departamento Penitenciário Brasileiro. Mulheres encarceradas: diagnóstico nacional. Brasília (DF): Ministério da Justiça; 2008.

11. França AMB, Silva JMO. Maternity in prison situation. Rev. baiana enferm. [Internet] 2015 [cited 2018 Feb 20]; 29(4):411-420. Available from em: https://portalseer.ufba.br/index.php/enfermagem/article/view/14026/pdf_22

12. Pimentel IS, Carvalho LFS, Carvalho SN, Carvalho CMS. Perception of women deprived of liberty health assistance of about the prison system R. Interd. [Internet] 2015 [cited 2018 Feb 20]; 8(4):109-19. Available from: https://revistainterdisciplinar.uninovafapi.edu.br/index.php/revinter/article/view/659/pdf_268

13. Silva PAS, Gomes LA, Amorim-Gaudêncio C, Lima KPN, Medeiros LB, Nogueira JA. Syphilis in women coming out of the prison system: prevalence and associated factors. Rev Rene [Internet] 2018 [cited 2018 Jun 02]. 19:e3321. Available from: http://periodicos.ufc.br/rene/article/view/32669/72850

14. Costa LHR, Alves JP, Fonseca CEP, Costa FM, Fonseca FF. Gender in the context of sexual and reproductive rights of women deprived of liberty. Enfermería Global [Internet] 2016 [cited 2018 Feb 20]; 43:151-63. Available from: http://scielo.isciii.es/pdf/eg/v15n43/pt docencia1.pdf

15. Canazaro D, Argimon IIL. Characteristics, depressive symptoms, and associated factors in incarcerated women in the State of Rio Grande do Sul, Brazil. Cad. Saúde Pública [Online] 2010 [cited 2018 Feb 20]; 26(7):121-43. Available from: http://www.scielo.br/pdf/csp/v26n7/11.pdf

16. Lopes TC, Roseni P. Trajetórias de mulheres privadas de liberdade: práticas de cuidado no reconhecimento do direito à saúde no Centro de Referência de Gestantes de Minas Gerais. Physis Revista de Saúde Coletiva [Internet]. 2016 [cited 2018 Feb 20] 26(4):1193-212. Available from: http://www.scielo.br/pdf/physis/v26n4/1809-4481-physis-26-04-01193.pdf

17. Alves MJH, Pereira EV, Belém JM, Quirino GS, Maia ER, Alencar AMPG. Factors of risk in sexual and reproductive health of women prisoners: integrative review. Rev baiana enferm [Internet] 2017 [cited 2018 Jun 02]; 31(1):e16241. Available from: https://portalseer.ufba.br/index.php/enfermagem/article/view/16241/14149

18. Ayres JRCM, França Júnior I, Calazans GJ, Saletti Filho HC. O conceito de vulnerabilidade e as práticas de saúde: novas perspectivas e desafios. In: Czeresnia D, Freitas CM, organizadores. Promoção da saúde - conceitos, desafios, tendências. 2 ed. Rev. Amp. Rio de Janeiro: Fiocruz; 2009. p. 117-39.

19. Ayres JRCM, Carvalho YM, Nasser MA, Saltão RM, Mendes VM. Ways of comprehensiveness: adolescentes and young adults in Primary Healthcare. Interface (Botucatu) 2012 [cited 2018 Feb 20]; 16(40):67-82. Available from: http://www.scielosp.org/scielo.php?script=sci_arttext\&pid=S1414-32832012000100006\&lng=en\&nrm=iso 
20. Ayres JRCM, Paiva V, França Júnior I, Gravato N, Lacerda R, Negra MD, et al. Vulnerability, human rights, and comprehensive health care needs of young people living with hiv/AIDS. Am. J. Public Health; 2006; 96:1001-6.

21. Ayres JRCM, França Júnior IF, Calazans GJ, Saletti Filho HC. Vulnerabilidade e prevenção em tempos de AIDS. In: Barbosa RM, Parker RG, organizadores. Sexualidade pelo avesso: direitos, identidade e poder. Rio de Janeiro: Relumé-Dumará/ABIA/IMSUERJ; 1999. p. 59-72.

22. De-La-Torre-Ugarte-Guanilo MC. Construção e validação de marcadores da vulnerabilidade de mulheres às DST/HIV na atenção básica à saúde [tese de doutorado]. São Paulo: Universidade de São Paulo; 2012.

23. Bertaux D. Narrativas de vida: a pesquisa e seus métodos. Tradução de Zuleide Alves Cardoso Cavalcante, Denise Maria Gurgel Lavallée. 2a ed. São Paulo: Paulus; 2010.

24. Sisdepen. Sistema de Informação do Departamento Penitenciário Nacional. Tribunal de Justiça. Bahia; 2019.

25. Conselho Nacional de Saúde (Br). Resolução no 466/2012 sobre pesquisa envolvendo seres humanos. Diário Oficial da União. Brasilia, DF, 13 jun. de 2013. Seção 1, p59.

26. Sgarbi RV, Carbone Ada S, Paião DS, Lemos EF, Simionatto S, Puga MA et al. A cross-sectional survey of HIV Testing and prevalence in twelve Brazilian Correctional Facilities. PLOS ONE. [internet] 2015 [cited 2018 Jun 02]. 10(10): 1-12. Available from: https://journals.plos.org/plosone/article?id=10.1371/journal.pone.0139487

27. Garaycochea MC, Pino R, Chávez I, Portilla JL, Miraval ML, Arguedas E, et al. Infecciones de transmisión sexual em mujeres de um establecimiento penitenciario de Lima, Perú. Rev Peru Med Exp. Salud Publica. 2013; 30(3):423-7.

28. Fernandes MA, Bezerra MM, Moura FMJSP, Alencar NES, Lima FFF, Castro AED. Sexually transmitted infections and the experiences of women in situations of imprisonment. Rev enferm UERJ [Internet] 2016 [cited 2018 Jun 02]. 24(6):e27774. DOI: http://dx.doi.org/10.12957/reuerj.2016.27774

29. Silva M. Saúde penitenciária no Brasil: plano e política. Brasília (DF): Verbena; 2015.

30. Coutinho FMM, Mourão PM, Costa PR, Lopes TC. O cuidado de mulheres privadas de liberdade e seus filhos: percursos e mediações necessárias de gestores e trabalhadores para a garantia do direito à saúde dessa população. In: Pinheiro R, Gerhardt $T E$, Silva Junior AG, Di Leo PF, Ponce MV, organizadores. Cultura do cuidado e o cuidado na cultura: dilemas, desafios e avanços para a efetivação da integralidade em saúde no MERCOSUL. Rio de Janeiro: IMS-UERJ; 2015. p. 199-210.

31. Ministério da Saúde (Br). Secretaria de Vigilância em Saúde. Guia de Vigilância em Saúde [Internet]. Brasília (DF): Ministério da Saúde, 2016.

32. Mann J, Tarantola DJM, Netter TW. A AIDS no mundo. Rio de Janeiro: Relume-Dumará; 1993.

33. Spindola T, Braga RMO, Marques SC, Formozo GA, Cecilio HPM, Oliveira DC. The self-protection against HIV for nursing professionals: study of social representations. Rev. enferm. UERJ [Internet] 2018 [cited 2018 Dec 30]; 26:e34277. DOI: https://doi.org/10.12957/reuerj.2018.34277

34. Gagnon, JH. Uma interpretação do desejo. Rio de Janeiro: Garamond; 2006. 